\title{
Selection of Statistical Features Based on Mutual Information for Classification of Human Coding and Non-coding DNA Sequences
}

\author{
Alan Wee-Chung Liew ${ }^{1}$, Yonghui $\mathrm{Wu}^{1}$ and Hong $\mathrm{Yan}^{1,2}$ \\ ${ }^{1}$ Department of Computer Engineering and Information Technology \\ 83 Tat Chee Avenue, City University of Hong Kong, Kowloon, Hong Kong \\ ${ }^{2}$ School of Electrical and Information Engineering \\ University of Sydney, NSW 2006, Australia
}

\begin{abstract}
The classification of human gene sequences into exons and introns is an important but difficult problem. We study the discriminative power of various statistical features (22 in total) in term of their mutual information (MI). By performing correlation analysis, we are able to identify a set of features that has high MI value while at the same time is complementary in their information content. Using the set of features, which consists of the three $S Z$ features, the AMI feature, and the first stop codon feature, we are able to achieve classification accuracy as high as $92 \%$.
\end{abstract}

\section{Introduction}

Gene prediction and the classification of coding and non-coding DNA sequences are unsolved and popular research problems in bioinformatics. During the past twenty years, numerous advance gene-finding algorithms have been developed and several review papers haven been published [1-3]. Although powerful machine learning algorithms are used in these algorithms, their performances are highly dependent on the coding statistics that are used to characterize the sequence. Many such statistics have been proposed in the past, for example: codon usage bias [4], base compositional bias between codon positions [5] and periodicity in base occurrence [6]. The use of a good set of coding statistics can directly impact the classification accuracy. Therefore, the selection of a set of optimal features for classification of coding and non-coding sequences is a very important problem.

Although many coding statistics have been proposed, a systematic comparison of their performance is still lacking. Such study would provide a useful guideline to the choice of good statistical features in a recognition algorithm. Recognition of coding regions or ORFs in human genome based on coding statistics is a difficult problem since the average length of exons of vertebrate gene is only $137 \mathrm{bp}$ [7]. Although good accuracy can be achieved in the recognition of coding and non-coding regions in yeast genome [8], statistical features alone are generally not sufficient to identify human exons due to their limited average length. The use of additional splicing signal such as stop codons results in better prediction power [9].

This paper reports our study on several statistical features that are often used for coding and non-coding DNA sequence classification. The features were compared based on their information content. A total of 22 features, divided into 6 groups, are analyzed. They are:

- Two ATG triplet features

- Two Stop-Codon features

- Three asymmetric features of nucleotides distribution at the three triplet positions

- $\quad$ Nine Z-Curve features

- $\quad$ Three Simple Z-Curve (SZ) features

- Three periodic features of correlations between nucleotide positions

\section{Methods}

We use the Human exon and intron datasets from http://bit.uq.edu.au/altExtron/. We extracted 1500 human exons and 1500 human introns. Their lengths are all chosen to be less than $140 \mathrm{bp}$. Although human introns can be potentially very long, short introns were selected since they are more easily confused with exons and also to avoid introducing any bias in recognition due to length. The exons and introns are used as positive and negative samples, respectively.

Based on the sequence information they contained, the 22 features can be classified into 6 groups:

1. Two ATG triplet features.

- Let the total number of the triplet ATG contained in all three frames in a sequence be denoted by $n$. The number of frames containing the triplet ATG in a sequence is denoted by $K$, i.e., $K=0,1,2,3$. The ATG triplet statistic is defined by: $f_{1}=\left(1+K^{2}\right) \times n$

- $f_{2}=N_{\mathrm{ATG}}$, where $N_{\mathrm{ATG}}$ is the number of triplet ATG in the sequence.

2. Two stop codon features.

The number of triplets TAA, TAG, and TGA occurring in each frame of the sequence is counted.

- As for $f_{1}$, the stop codon feature is defined by $f_{3}=\left(1+K^{2}\right) \times n$. This feature has been used in [9].

- $f_{4}=\max \left(N_{T A A}, N_{T A G}, N_{T G A}\right)$

3. Three asymmetric features of distribution of nucleotides at the three triplet positions.

- The Position Asymmetry (PA) of the sequence [3].

Let $f(b, r)$ be the (relative) frequency of nucleotide $b$ at triplet position $r$. Let $f(b)=\sum_{r=1}^{3}(f(b, r)) / 3$ be the 
average frequency of nucleotide $b$ at the three triplet positions, and define the asymmetry in the distribution of nucleotide $b$ as the variance of this frequency, i.e., $\operatorname{asym}(b)=\sum_{i=1}^{3}(f(b, i)-f(b))^{2}$, the PA of the sequence is defined as:

$f_{5}=P A=\operatorname{asym}(A)+\operatorname{asym}(C)+\operatorname{asym}(G)+\operatorname{asym}(T)$

- One Purine feature.

It is well known that the predominant bases at the first codon position are purines and this fact is independent of species [3], whereas bases in non-coding regions tend to be randomly distributed. The occurrence frequencies of purines in the three reading frames are denoted by $\left(a_{i}+g_{i}\right), \quad i=1,2,3$, and the purine feature is defined as: $f_{6}=\max _{i}\left(a_{i}+g_{i}\right), \quad i=1,2,3$

- One Pyrimidine feature.

It is well known that the predominant bases at the third codon position are pyrimidines and this fact is independent of species [3]. The occurrence frequencies of pyrimidines in the three frames are denoted by $\left(c_{i}+t_{i}\right), \quad i=1,2,3$. The pyrimidine feature is defined as: $f_{7}=\max _{i}\left(c_{i}+t_{i}\right), \quad i=1,2,3$

4. Nine Z-Curve features $[10,11]$.

The Z-Curve features are based on the differences of single nucleotide frequencies at the three codon positions between the protein coding ORFs and the non-coding ones. The frequencies of bases A, C, G and $\mathrm{T}$ occurring in an ORF or a fragment of DNA sequence with bases at positions $1,4,7, \ldots ; 2,5,8, \ldots$; and 3,6 , $9, \ldots$, are denoted by $a_{1}, c_{1}, g_{1}, t_{1} ; a_{2}, c_{2}, g_{2}, t_{2} ; a_{3}, c_{3}, g_{3}$, $t_{3}$, respectively. They are actually the frequencies of bases at the first, second and third codon position. Let $x_{1}, y_{1}, z_{1} ; x_{2}, y_{2}, z_{2} ; x_{3}, y_{3}, z_{3}$ be given by

$$
\left\{\begin{array}{l}
x_{i}=\left(a_{i}+g_{i}\right)-\left(c_{i}+t_{i}\right) \\
y_{i}=\left(a_{i}+c_{i}\right)-\left(g_{i}+t_{i}\right), \quad i=1,2,3 \\
z_{i}=\left(a_{i}+t_{i}\right)-\left(g_{i}+c_{i}\right)
\end{array}\right.
$$

The nine Z-Curve features, $f_{8}$ to $f_{16}$, are defined by

$$
\begin{gathered}
f_{8}=x_{1}, f_{9}=y_{1}, f_{10}=z_{1} \\
f_{11}=x_{2}, f_{12}=y_{2}, f_{13}=z_{2} \\
f_{14}=x_{3}, f_{15}=y_{3}, f_{16}=z_{3}
\end{gathered}
$$

5. Three Simple Z-Curve (SZ) features [12].

The SZ features, $f_{17}$ to $f_{19}$, are defined by

$$
\begin{aligned}
& f_{17}=\max _{i}\left[\left(a_{i}+g_{i}\right)-\left(c_{i}+t_{i}\right)\right] \\
& f_{18}=\max _{i}\left[\left(a_{i}+c_{i}\right)-\left(g_{i}+t_{i}\right)\right], \quad i=1,2,3 \\
& f_{19}=\max _{i}\left[\left(a_{i}+t_{i}\right)-\left(g_{i}+c_{i}\right)\right]
\end{aligned}
$$

6. Three periodic correlation features between nucleotide positions $[5,3]$.

- Periodic Asymmetry Index (PAI).

Given a sequence, Konopka [13] considers three distinct probabilities, the probability $P_{i n}$ of finding pairs of the same nucleotide at distances $k=2,5,8, \ldots$, the probability $P_{\text {out }}^{1}$ of finding pairs of the same nucleotide at distances $k=0,3,6, \ldots$, and the probability $P_{\text {out }}^{2}$ of finding pairs of the same nucleotide at distances $k=1,4,7, \ldots$. Because of the 3-base periodic pattern, $P_{i n}$ will be larger than the other two probabilities in coding regions. In non-coding regions the three probabilities will be similar. The tendency to cluster homogeneous di-nucleotides in a 3-base periodic pattern can be measured by the PAI,

$$
f_{20}=P A I=\frac{\max \left(P_{\text {in }}, P_{\text {out }}^{1}, P_{\text {out }}^{2}\right)}{\min \left(P_{\text {in }}, P_{\text {out }}^{1}, P_{\text {out }}^{2}\right)}
$$

- Average Mutual Information (AMI) [14].

The correlation $\left(\rho_{i j}(k)\right)$ between nucleotide $i$ and nucleotide $j$ at a distance of $k$ nucleotides can be calculated as $\rho_{i j}(k)=p_{i j}(k)-p_{i} p_{j}$, where $p_{i}$ and $p_{j}$ are the probabilities of nucleotides $i$ and $j$ in the sequence, and $p_{i j}(k)$ is the probability in the sequence of the pair of nucleotides $i$ and $j$ at a distance of $k$ nucleotides. Thus, for each distance $k$, sixteen different individual correlations can be calculated. A measure that summarizes all individual correlations at a given distance $k$ is the mutual information function,

$$
I(k)=\sum_{i, j \in\{A, C, G, T\}} P_{i j}(k) \log _{2}\left(\frac{P_{i j}(k)}{P_{i} P_{j}}\right)
$$

The mutual information $I(k)$ quantifies the amount of information that can be obtained from one nucleotide about another nucleotide at a distance $k$. In coding DNA, $I(k)$ oscillates between two values, while in non-coding DNA, $I(k)$ is rather flat. Herzel and Grosse [14] called the two values between which $I(k)$ oscillates in coding DNA the in-frame mutual information $I_{\text {in }}$ at distances $k$ $=2,5,8, \ldots$, and the out-of-frame mutual information $I_{\text {out }}$ at $k=4,5,7,8, \ldots$ In order to reduce the pair of numbers $I_{\text {in }}$ and $I_{\text {out }}$ to a single quantity, they compute the average mutual information (AMI) as

$$
f_{21}=A M I=\frac{I_{\text {in }}+2 I_{\text {out }}}{3}
$$

- Fourier spectral feature [6].

Periodic correlations in DNA sequences can also be examined by means of Fourier analysis. DNA coding regions reveal the characteristic 3-base periodicity which shows up as a distinct peak at frequency $f=1 / 3$. No such peak is apparent for non-coding sequences. We defined the Fourier spectrum feature $f_{22}$ as

$$
f_{22}=\frac{f_{1 / 3}}{\frac{1}{2 k+1} \sum_{j=i-k}^{j=i+k} f_{j}}
$$

Altogether 22 statistical features are compared. Their discriminative power for coding/non-coding sequences is compared using the information-theoretic measure called mutual information, which essentially measures the 
information the feature tells us about the two classes (i.e., coding vs non-coding). The mutual information of the $j$-th feature with respect to the class $\omega_{i}$ is given by

$$
G_{j}=\sum_{i=1}^{m} \sum_{k=1}^{v_{j}} p\left(\omega_{i}, f_{j}(k)\right) \log \frac{p\left(\omega_{i} \mid f_{j}(k)\right)}{p\left(\omega_{i}\right)}
$$

where each feature $f_{j}$ has $v_{j}$ discrete values which are obtained by histogramming. The mutual information $G_{j}$ measures the information that $f_{j}$ tells us about the class label. Equation (13) can be written as

$G_{j}=\sum_{i=1}^{m} \sum_{k=1}^{v_{j}} p\left(\omega_{i}\right) p\left(f_{j}(k) \mid \omega_{i}\right) \log \frac{p\left(f_{j}(k) \mid \omega_{i}\right)}{p\left(f_{j}(k)\right)}$

For 2 class coding/non-coding classification, $m=2$. We set the prior probabilities $p\left(\omega_{i}\right)$ to be equal for both classes.

\section{Results and Discussion}

From Table 1, we see that features that measure the 3 -periodicity $\left(f_{5}, f_{20}, f_{21}\right.$, and $\left.f_{22}\right)$ generally have higher MI. The two SZ features $\left(f_{17}, f_{19}\right)$ also have higher than average MI. Of the nine Z-Curve features, only $f_{13}$ has MI that is above the mean value, indicating that this set of features is not very discriminative for human exon/intron classification. To provide a baseline comparison, the MI of a set of samples randomly selected from the exon database and a set of samples randomly selected from the intron database are computed and they are all below 0.02 .

To obtain good classification result, the set of features used should have high MI value while at the same time be complementary in their information content. In other word, we look for a set of features that have high MI value but are as uncorrelated to each other as possible. To see how the different sets of features relate to each others, we perform a correlation analysis of the 22 features by

$$
r_{x y}=\frac{\sum_{i=1}^{n}\left(x_{i}-\bar{x}\right)\left(y_{i}-\bar{y}\right)}{\sqrt{\sum_{i=1}^{n}\left(x_{i}-\bar{x}\right)^{2} \sum_{i=1}^{n}\left(y_{i}-\bar{y}\right)^{2}}}
$$

where $x_{i}$ and $y_{i}$ are the two features to be correlated, $\bar{x}$ and $\bar{y}$ are their mean values, and $n$ is the number of samples in the dataset. The correlation analysis gives the following results, where, only features that are highly correlated (with correlation value $>0.4$ ) are listed:

ATG triplet feature 1: ATG triplet feature $2(0.8548)$

Stop codon 1: stop codon 2 (0.7249)

PA: AMI(0.8995), PAI(0.7985), FFT(0.5526)

Purines: $\mathrm{SZ}_{1}(0.6812)$, Pyrimidine $(0.4949), Z_{1}(0.432)$

Pyrimidines: Purines $(0.4949)$

$\mathrm{Z}_{1}: \mathrm{SZ}_{1}(0.7003)$, Purines $(0.4320)$

$\mathrm{Z}_{2}: \mathrm{SZ}_{2}(0.6217)$

$\mathrm{Z}_{3}: \mathrm{SZ}_{3}(0.5846)$

$\mathrm{Z}_{4}: \mathrm{SZ}_{1}(0.5956)$

$\mathrm{Z}_{5}: \mathrm{SZ}_{2}(0.5964)$
$\mathrm{Z}_{6}: \mathrm{SZ}_{3}(0.6466)$

$\mathrm{Z}_{7}: \mathrm{SZ}_{1}(0.6004)$

$\mathrm{Z}_{8}: \mathrm{SZ}_{2}(0.5863)$

$Z_{9}: \mathrm{SZ}_{3}(0.5604)$

$S Z_{1}: Z_{1}(0.7003)$, Purines $(0.6812), Z_{7}(0.6004), Z_{4}(0.5956)$

$\mathrm{SZ}_{2}: \mathrm{Z}_{2}(0.6217), \mathrm{Z}_{5}(0.5964), \mathrm{Z}_{8}(0.5863)$

$\mathrm{SZ}_{3}: \mathrm{Z}_{6}(0.6466), \mathrm{Z}_{3}(0.5846), \mathrm{Z}_{9}(0.5604)$

PAI: AMI $(0.8247), \operatorname{PA}(0.7985)$, FFT $(0.6600)$

AMI: PA(0.8995), PAI(0.8247), FFT(0.4385)

FFT: $\operatorname{PAI}(0.6600), \operatorname{PA}(0.5526), \operatorname{AMI}(0.4385)$

Based on the correlation analysis, we can grouped the 22 features into 4 classes: (1) class 1 consists of the nine Z-Curve features, three SZ features, Purines, and the Pyrimidines feature, (2) class 2 consists of the two ATG triplet features, (3) class 3 consists of the two stop codon, and finally, (4) class 4 consists of the PA, PAI, AMI and FFT features. By examining the correlation values and the MI values, we can select features that are effective for the classification task.

For the class 1 features, we see that $\mathrm{SZ}_{1}$ is highly correlated with $Z_{1}$, Purines (which in turn is highly correlated with Pyrimidines), $Z_{7}$, and $Z_{4}$. Among the five features, $\mathrm{SZ}_{1}$ has the highest MI. So, $\mathrm{SZ}_{1}$ is selected as a feature to be used in the classification task. Similarly, $\mathrm{SZ}_{2}$ and $\mathrm{SZ}_{3}$ are also selected. For the two class 2 features, their MIs are of low value and neither feature is selected. For the class 3 features, the first stop codon feature is selected. For the class 4 features, the AMI feature is selected.

We perform classification experiments using the five selected features. The classifier we used is the K-Nearest-Neighbors (KNN) classifier. Unlike the Fisher Discriminant classifier, KNN classifier does not require the decision surface to be linear and tends to produce superior results for the exon-intron classification task [12]. The 1500 exons and 1500 introns in the dataset are randomly divided into two parts. Part 1 is taken as the training set and part 2 as the testing set. The sensitivity, specificity and accuracy of the algorithm based on part 2 are calculated. Then, the procedure is repeated by reversing the roles of the two parts. The classification is performed three times. For the first time, part 1 contains 750 exons and 750 introns, and part 2 contains 750 exons and 750 introns. For the second and third times, the partition of the two parts becomes $500+1000$ and $250+1250$, respectively. The sensitivity $(S n)$ and specificity $(S p)$ are as defined in [15], where $S n$ is the proportion of coding nucleotides that have been correctly classified as coding, and $S p$ is the proportion of non-coding nucleotides that have been correctly classified as non-coding. The average sensitivity, specificity and accuracy over the six-fold cross-validation test are calculated and listed in Table 2.

The classification results in Table 2 are better than the classification results using a different combination of feature sets. For example, the feature set $\left\{\mathrm{SZ}_{1}, \mathrm{SZ}_{2}, \mathrm{SZ}_{3}\right.$, AMI, PA, PAI, FFT, first stop codon feature $\}$ gives a 
maximum classification accuracy of 0.905 , with sensitivity and specificity all lower than the results of Table 2, even though a larger set of features is used. The best classification results using the nine Z-Curve features proposed by Zhang $[10,11]$ gives a maximum classification accuracy of only 0.884 .

Table 1. Comparison of MI of 22 statistical features

\begin{tabular}{|c|c|c|}
\hline \multicolumn{2}{|c|}{ ATG triplet feature $1\left(f_{1}\right)$} & 0.0378 \\
\hline \multicolumn{2}{|r|}{ ATG triplet feature $2\left(f_{2}\right)$} & 0.0550 \\
\hline \multicolumn{2}{|r|}{ Stop Codon feature $1\left(f_{3}\right)$} & 0.1000 \\
\hline \multicolumn{2}{|r|}{ Stop Codon feature $2\left(f_{4}\right)$} & 0.0110 \\
\hline \multicolumn{2}{|r|}{ Position Asymmetry, PA $\left(f_{5}\right)$} & 0.3073 \\
\hline \multicolumn{2}{|r|}{ First Codon (Purines) $\left(f_{6}\right)$} & 0.0629 \\
\hline \multicolumn{2}{|r|}{ Third Codon (pyrimidines) $\left(f_{7}\right)$} & 0.0546 \\
\hline \multirow{9}{*}{$\mathrm{Z}$} & $\mathrm{Z}_{1}:\left(\mathrm{a}_{1}+\mathrm{g}_{1}\right)-\left(\mathrm{c}_{1}+\mathrm{t}_{1}\right)\left(f_{8}\right)$ & 0.0451 \\
\hline & $\mathrm{Z}_{2}:\left(\mathrm{a}_{1}+\mathrm{c}_{1}\right)-\left(\mathrm{g}_{1}+\mathrm{t}_{1}\right)\left(f_{9}\right)$ & 0.0417 \\
\hline & $\mathrm{Z}_{3}:\left(\mathrm{a}_{1}+\mathrm{t}_{1}\right)-\left(\mathrm{c}_{1}+\mathrm{g}_{1}\right)\left(f_{10}\right)$ & 0.0826 \\
\hline & $\mathrm{Z}_{4}:\left(\mathrm{a}_{2}+\mathrm{g}_{2}\right)-\left(\mathrm{c}_{2}+\mathrm{t}_{2}\right)\left(f_{11}\right)$ & 0.0862 \\
\hline & $\mathrm{Z}_{5}:\left(\mathrm{a}_{2}+\mathrm{c}_{2}\right)-\left(\mathrm{g}_{2}+\mathrm{t}_{2}\right)\left(f_{12}\right)$ & 0.0839 \\
\hline & $\mathrm{Z}_{6}:\left(\mathrm{a}_{2}+\mathrm{t}_{2}\right)-\left(\mathrm{c}_{2}+\mathrm{g}_{2}\right)\left(f_{13}\right)$ & 0.1111 \\
\hline & $\mathrm{Z}_{7}:\left(\mathrm{a}_{3}+\mathrm{g}_{3}\right)-\left(\mathrm{c}_{3}+\mathrm{t}_{3}\right)\left(f_{14}\right)$ & 0.0388 \\
\hline & $\mathrm{Z}_{8}:\left(\mathrm{a}_{3}+\mathrm{c}_{3}\right)-\left(\mathrm{g}_{3}+\mathrm{t}_{3}\right)\left(f_{15}\right)$ & 0.0215 \\
\hline & $\mathrm{Z}_{9}:\left(\mathrm{a}_{3}+\mathrm{t}_{3}\right)-\left(\mathrm{c}_{3}+\mathrm{g}_{3}\right)\left(f_{16}\right)$ & 0.0683 \\
\hline \multirow{3}{*}{$\mathrm{SZ}$} & $\mathrm{SZ}_{1}: \max _{i}\left[\left(a_{i}+g_{i}\right)-\left(c_{i}+t_{i}\right)\right]\left(f_{17}\right)$ & 0.1406 \\
\hline & $\mathrm{SZ}_{2}: \max _{i}\left[\left(a_{i}+c_{i}\right)-\left(g_{i}+t_{i}\right)\right]\left(f_{18}\right)$ & 0.0985 \\
\hline & $\mathrm{SZ}_{3}: \max _{i}\left[\left(a_{i}+t_{i}\right)-\left(g_{i}+c_{i}\right)\right]\left(f_{19}\right)$ & 0.2977 \\
\hline \multicolumn{2}{|r|}{ PAI $\left(f_{20}\right)$} & 0.1478 \\
\hline \multicolumn{2}{|r|}{$\operatorname{AMI}\left(f_{21}\right)$} & 0.1760 \\
\hline \multicolumn{2}{|r|}{ FFT $\left(f_{22}\right)$} & 0.1182 \\
\hline \multicolumn{2}{|r|}{ Mean value } & 0.0994 \\
\hline
\end{tabular}

Table 2. The average sensitivity ( $\mathrm{Sn}$ ), specificity (Sp), and accuracy (Ac) for human Exon/Intron classification based on SZ1, SZ2, SZ3, AMI and the first stop codon feature

\begin{tabular}{|c|c|c|c|c|}
\hline \# Training & \# Testing & Sn & Sp & Ac \\
\hline 250 & 1250 & 0.8922 & 0.8940 & 0.8931 \\
\hline 500 & 1000 & 0.8900 & 0.9105 & 0.9002 \\
\hline 750 & 750 & 0.8990 & 0.9090 & 0.9040 \\
\hline 1000 & 500 & 0.9105 & 0.9040 & 0.9073 \\
\hline 1250 & 250 & 0.9120 & 0.9270 & 0.9195 \\
\hline
\end{tabular}

\section{Conclusion}

Various statistical features have been proposed for the classification of exon/intron. However, not all features are equally effective for the classification task. Choosing a set of highly discriminative features could significantly improve the classification accuracy. In this work, we study the various statistical features for human exon/intron classification, which is difficult due to the average short exon length. By computing the mutual information, we are able to quantify the effectiveness of the features. Together with correlation analysis of the features, we are able to select a small set of features, which consists of the three SZ features, the AMI feature, and the first stop codon feature, that gives a classification accuracy of up to $92 \%$.

\section{Acknowledgment}

This work is supported by a CityU interdisciplinary grant (project 9010003).

\section{References}

[1] Fickett, J.W. and Tung, C.S. (1992). Assessment of protein coding measures. Nucleic Acids Research 20:6641-6450.

[2] Fickett, J.W. (1996). Finding genes by computer: the state of the art. Trends Genet. 12: 316-320.

[3] Guigo, R. (1999). DNA Composition, Codon Usage and Exon Prediction. In M. Bishop, editor: Genetic Databases. Academic Press. 53-80.

[4] R. Staden and A.D. McLachlan (1982). Codon preference and its use in identifying protein coding regions in long DNA sequences. Nucleic Acids Research. 10, 141-156.

[5] J.W. Fickett (1982). Recognition of protein coding regions in DNA sequences. Nucleic Acids Research. 10, 5303-5318.

[6] S. Tiwari, S. Ramachandran, A. Bhattacharya, S. Bhattacharya, and R. Ramaswamy (1997). Prediction of probable genes by Fourier analysis of genomic sequences. Computer Applications in Bioscience. 13, 263-270.

[7] J. D. Hawkins (1988). A survey on intron and exon lengths. Nucleic Acids Research. 16, 9893-9908.

[8] C. T. Zhang and J. Wang (2000). Recognition of protein coding genes in the Yeast genome at better than $95 \%$ accuracy based on the $\mathrm{Z}$ curve. Nucleic Acids Research. 28, 2804-2814.

[9] Y. Wang, C.T. Zhang, and P. Dong (2002). Recognizing shorter coding regions of human genes based on the statistics of stop codons. Biopolymers. 63(3), 207-216.

[10] Zhang, R. and Zhang, C.T. (1994). Z Curves, an Intuitive Tool for Visualizing and Analyzing DNA sequences. Journal Biomolecular Structure Dynamics. 11:767-782.

[11] Zhang, C.T. (1997). A symmetrical theory of DNA sequences and its application. J. Theoretical Biol. 187: 297-306. [12] Wu Y., Liew A.W.C., Yan H., and Yang M. (2003). Classification of Short Human Exons and Introns based on Statistical Features. Physical Review E. 67(6): Art. No. 061916. [13] Konopka, A. K. (1994). Structure and Methods: VI. Human Genome Initiative and DNA Recombination, chapter Towards Mapping Functional Domains in Indiscriminantly Sequenced Nucleic Acids: A Computational Approach. Adenine Press, Guilderland, New York.

[14] Herzel, H. and Grosse, I. (1995). Measuring correlations in symbol sequences. Physica A 216:518-542.

[15] Burset, M. and Guigo, R. (1996). Evaluation of Gene Structure Prediction Programs. Genomics.34: 353-367. 